\title{
Distribuição Geográfica e Diversidade Morfológica de Culturas de Penicillium sclerotigenum em Inhames no Brasil
}

\author{
Idjane S. Oliveiraํㅜ, Edna Dora M.N. Luz ${ }^{2}$ Romero M. Moura ${ }^{3}$ \& Leonor C. Maia ${ }^{4}$ \\ ${ }^{1}$ Departamento de Ciências Biológicas, Universidade Estadual de Santa Cruz, CEP 45600-000, Ilhéus, BA, Brasil; ${ }^{2}$ Seção \\ de Fitopatologia, CEPEC, CEPLAC, CEP 45650-000, Itabuna, BA, Brasil; ${ }^{3}$ Departamento de Agronomia, Área de \\ Fitossanidade, Universidade Federal Rural de Pernambuco, CEP 52171-900, Recife, PE, Brasil; ${ }^{4}$ Departamento de \\ Micologia, Universidade Federal de Pernambuco, CEP 50670-420, Recife, PE, Brasil, e-mail: idjaneoliveira@yahoo.com
}

Autor para correspondência: Idjane S. Oliveira

OLIVEIRA, I.S., LUZ, E.D.M.N., MOURA, R.M. \& MAIA, L.C. Distribuição geográfica e diversidade morfológica de culturas de Penicillium sclerotigenum em inhames no Brasil. Fitopatologia Brasileira 32:131-136. 2007.

\section{RESUMO}

Foi realizado um levantamento de ocorrência da podridão-verde do inhame em diferentes Estados brasileiros, com análise dos tipos de colônia do patógeno, em 50 isolados de Penicillium obtidos para identificação. Os isolados foram originados de túberas infectadas das duas espécies de inhame Dioscorea cayennensis e D. alata. Os resultados foram obtidos mediante técnicas específicas de identificação de espécies do gênero Penicillium e práticas laboratoriais rotineiras de Fitopatologia. Constatou-se que a doença ocorria em todos os Estados estudados, sendo a doença sempre causada por Penicillium sclerotigenum, único organismo em constante associação com a doença. Os isolados do fungo apresentaram diversidade na morfologia das colônias, independentemente da espécie de Dioscorea e local de coleta da amostra.

Palavras-chave adicionais: podridão-verde do inhame, Dioscorea cayennensis, Dioscorea alata, doenças do inhame.

\begin{abstract}
Geographic distribution and morphologic diversity of cultures of Penicillium sclerotigenum of yams in Brazil

A survey was carried out to determine the occurrence of soft-rot of yam in different states of Brazil. Fungus isolates were taken from diseased tubers of two edible yam species, Dioscorea cayennensis and D. alata. Observations were carried out to identify the 50 isolates of Penicillium obtained using specific techniques for the genus Penicillium taxonomy and routine laboratory techniques of plant pathology. It was concluded that the disease occurred in all surveyed states and Penicillium sclerotigenum was the only Penicillium species in constant association with the disease. The pathogen isolates presented high colony variation, independently of the yam species and collecting place.
\end{abstract}

Additional keywords: Green-rot of yams, Dioscorea cayennensis, Dioscorea alata, yams diseases.

\section{INTRODUÇÃO}

A cultura do inhame (Dioscorea spp.) tem sido explorada comercialmente nas regiões sudeste e nordeste do Brasil, há muitas décadas, e anualmente novos grandes projetos têm sido iniciados com financiamento por agências de fomento. Os estados com maiores taxas de produção no Nordeste são Paraíba, principal produtor, Pernambuco, Bahia, Alagoas e Maranhão. A grande maioria dos plantios ainda constitui uma atividade agrícola tipicamente familiar, mas que gera renda e trabalho, empregando, em média, 1,25 homem/hectare/ano (Santos, 2002). Além dos empregos diretos, a cadeia produtiva do inhame envolve outros setores como armazenamento, transporte e comercialização. Dessa forma, pode-se afirmar que a cultura do inhame apresenta grande importância econômica e social para o desenvolvimento daquela região.

Parte da Tese de Doutorado da primeira autora. Universidade Federal de Pernambuco. Recife PE. 2006.
Entre os problemas fitossanitários mais importantes que podem restringir sobretudo o comércio exportador do inhame da Costa, encontra-se a podridão-verde, doença do tipo pós-colheita, de alta severidade, que incide sobre túberas comerciais e sementes dos inhames da Costa e São Tomé (Moura, 2005). O agente etiológico é o fungo Penicillium sclerotigenum, descrito inicialmente no Japão por Yamamoto et al., 1955, parasitando inhame chinês (Dioscorea batatas Dec). No Brasil, essa doença foi assinalada por Moura et al. (1976) em Pernambuco, ocasião em que foi criada a denominação podridão-verde, apresentada a sintomatologia e etiologia da doença, relatadas as condições de predisposição e feita breve descrição do patógeno, fundamentando-se os autores em isolado único.

O primeiro sintoma perceptivo da podridão-verde é uma pequena mancha úmida, sempre associada a ferimento na túbera, originando uma lesão profunda, de coloração marrom, sobre a qual observa-se uma camada de micélio, de cor verde acinzentado, formada por conidióforos e conídios do fungo, quando as condições climáticas são favoráveis, tais como: temperatura e umidade altas. Em seguida, ao iniciar 
a saprogênese, $P$. sclerotigenum produz grande quantidade de esclerócios dentro dos tecidos colonizados, uma das principais características da espécie. A podridão progride rapidamente e no máximo em 20 dias toda a túbera fica comprometida (Santos, 1996). Ferimentos em túberas são freqüentes, provocados, principalmente, no ato da colheita, limpeza, armazenamento e transporte. A doença torna a túbera comercial imprópria para consumo e nas sementes a brotação é comprometida, ocasionando falhas no plantio (Moura et al., 1976).

São poucas as informações disponíveis na literatura mundial sobre a podridão-verde do inhame e $P$. sclerotigenum. Ogundana et al. (1970) mencionaram P. sclerotigenum entre outros fungos, como causadores de podridão do inhame na Nigéria sendo, na ocasião, revelada a espécie P. italicum Wehmer associada à doença. Adeniji (1970) reportou alta freqüência da ocorrência de $P$. oxalicum Currie \& Thom, causando podridão de túberas do inhame também na Nigéria. Essas espécies ainda não foram registradas ocorrendo em inhame no Brasil, onde não existem também dados sobre distribuição geográfica da doença nem informações sobre diversidade em tipos de colônia de isolados de $P$. sclerotigenum. Por outro lado, são inexistentes registros na literatura mundial de estudos envolvendo mais de oito isolados de $P$. sclerotigenum, não tendo sido relatada pelos autores de assinalamentos, nem pelos demais, até o momento, diversidade morfológica do patógeno, nem em tipos de colônias (Yamamoto et al., 1955; Moura et al., 1976; Samson \& Frisvad, 2004).

À luz de tais informações, os objetivos da presente pesquisa foram estudar ocorrência da podridão-verde do inhame em diferentes estados brasileiros, investigando-se distribuição geográfica da doença, prevalência de $P$. sclerotigenum como patógeno, bem como a sua diversidade morfológica.

\section{MATERIAL E MÉTODOS}

\section{Coleta de túberas comerciais de inhame}

A pesquisa teve início com a coleta de túberas comerciais de inhame da Costa e São Tomé, portadoras de sintomas da podridão-verde, em Centrais de Abastecimento, feiras livres e supermercados nos estados da Bahia, Paraíba, Pernambuco, Rio Grande do Norte, São Paulo, Rondônia e no Distrito Federal. Uma vez coletadas, as amostras eram acondicionadas em sacos plásticos individualizados, rotulados e transportados para laboratório para isolamento no mesmo dia.

\section{Isolamento de Penicillium}

Pequenos fragmentos retirados de margens de tecidos infectados naturalmente de túberas de inhame da Costa e São Tomé foram devidamente lavados com água e sabão e em seguida, procedido isolamento indireto para fungos fitopatogênicos, conforme descrito em Menezes \& Assis (2004). Para tanto, os fragmentos foram desinfestados superficialmente por meio de uma passagem em etanol a $50 \%$, seguindo-se outra em hipoclorito de sódio diluído 1:3 com água destilada, lavando-se, em seguida, em água destilada esterilizada. Após a desinfestação, os fragmentos foram semeados nos meios BDA
(Batata - Dextrose - Agar) e MEA (Blakeslee Malt Extract Autolysate - extrato de malte $3,0 \%$, peptona $0,1 \%$, glicose 2,0\%, $\mathrm{CuSO}_{4} \cdot 5 \mathrm{H}_{2} \mathrm{O}$ 0,0005\%, $\mathrm{ZnSO}_{4} \cdot 7 \mathrm{H}_{2} \mathrm{O}$ 0,001\%, Agar $2,0 \%)$. Obtidas culturas puras, os isolados foram catalogados, utilizando-se códigos referentes às siglas dos Estados de origem, em numeração seqüencial. Cada isolado era posteriormente submetido a testes de inoculação em três seções circulares de inhame da Costa ou São Tomé, de 1,5 cm de espessura, para confirmação da patogenicidade. Nesses testes, usaram-se câmaras úmidas, mantidas em laboratório por sete dias, e em seguida, procederam-se os reisolamentos.

\section{Identificação de isolados de Penicillium sp.}

A identificação dos isolados de Penicillium a nível de espécie foi realizada de acordo com a chave de classificação elaborada por Samson \& Frisvad (2004). Inicialmente, em condições assépticas, propágulos de cada isolado foram semeados em três pontos eqüidistantes, em placa de Petri, contendo cada um dos meios de cultura utilizados. Os meios usados e indicados para identificação de espécies do subgênero Penicillium, foram MEA; Czapek Yeast Autolysate $-\mathrm{NaNO}_{3} 0,3$ $\%$, extrato de levedura - Difco 0,5 \%, sacarose 3,0 \%, $\mathrm{K}_{2} \mathrm{HPO}_{4}$. $3 \mathrm{H}_{2} \mathrm{O} 0,13 \%, \mathrm{MgSO}_{4} \cdot 7 \mathrm{H}_{2} \mathrm{O}$ 0,05 \%, KCL 0,05 \%, $\mathrm{FeSO}_{4}$. $7 \mathrm{H}_{2} \mathrm{O} 0,001 \%$, $\mathrm{CuSO}_{4} \cdot 5 \mathrm{H}_{2} \mathrm{O} 0,0005 \%, \mathrm{ZnSO}_{4} \cdot 7 \mathrm{H}_{2} \mathrm{O} 0,001 \%$, Agar 1,5\%); Yeast Extract Sucrose - extrato de levedura 2,0\%, sacarose $15,0 \%$, $\mathrm{MgSO}_{4} .7 \mathrm{H}_{2} \mathrm{O} 0,05 \%$, $\mathrm{CuSO}_{4} \cdot 5 \mathrm{H}_{2} \mathrm{O} 0,0005 \%$, $\mathrm{ZnSO}_{4} \cdot 7 \mathrm{H}_{2} \mathrm{O} 0,001 \%$, Agar 2,0 \%); Creatine Sucrose - creatina $0,3 \%$, sacarose $3,0 \%, \mathrm{~K}_{3} \mathrm{PO}_{4} .7 \mathrm{H}_{2} \mathrm{O} 0,16 \%, \mathrm{MgSO}_{4} .7 \mathrm{H}_{2} \mathrm{O} 0,05$ $\%$, KCl 0,05 \%, $\mathrm{FeSO}_{4} .7 \mathrm{H}_{2} \mathrm{O} 0,001 \%$, $\mathrm{CuSO}_{4} .5 \mathrm{H}_{2} \mathrm{O} 0,0005$ $\%$, $\mathrm{ZnSO}_{4} \cdot 7 \mathrm{H}_{2} \mathrm{O}$ 0,001 \%, púrpura de bromocresol 0,005 \%, Agar 1,5\%) e meio Czapek Yeast Autolysate com $5 \%$ de NaCl. A temperatura de incubação dos isolados em todos os meios foi $25^{\circ} \mathrm{C}$, porém as placas de CYA foram também incubadas a $30^{\circ} \mathrm{C}$ em sala de incubação com refrigeração mantida por ar condicionado para as temperaturas determinadas. Os isolados cresceram em condições de luz ambiente, nas bancadas da sala de incubação por sete dias, com a exceção para os cultivados no meio CREA que foram avaliados aos sete e quatorze dias, quanto à produção ou não de ácido ciclopiazônico ou outros alcalóides relacionados, de acordo com Samson \& Frisvad (2004). Para completar a identificação da espécie, foram realizadas micrometrias em lâminas preparadas com azul de Amann, oriundas de colônias com sete dias de desenvolvimento em meio MEA. As estruturas taxonômicas do subgênero Penicillium avaliadas foram: comprimento e largura de conídio, fiálide, metula, ramo e esclerócio. Esta última por ser estrutura característica da espécie $P$. sclerotigenum. Vinte unidades de cada estrutura e de cada isolado foram medidas, a fim de obter um valor médio para cada isolado e ao final das medições obter um valor médio para cada estrutura taxonômica com base nos cinqüenta isolados, representando $P$. sclerotigenum no Brasil. Como complemento, observou-se a produção de esclerócios nos meios MEA e CYA, principal estrutura taxonômica diferenciadora da espécie P. sclerotigenum (Samson \& Frisvad, 2004). Todos os isolados foram preservados em subcultivos periódicos, em tubos de ensaio contendo meio CYA, em óleo 
mineral esterilizado, à temperatura ambiente e, também, em água destilada esterilizada, a $4{ }^{\circ} \mathrm{C}$ (Figueiredo, 2001).

\section{RESULTADOS E DISCUSSÃO}

A partir das amostras de túberas de inhame da Costa e São Tomé com sintomas de podridão-verde, coletadas em diferentes estados brasileiros, foi obtido um total de 50 isolados de Penicillium que, após identificação específica, foram depositados na Coleção de Culturas Fúngicas (Micoteca) do Departamento de Micologia da Universidade Federal de Pernambuco, cadastrada no CMI (Comnonwealth Mycological
Institute, Inglaterra) com a sigla URM (University Recife Mycology).

Em relação à distribuição geográfica, a podridão-verde foi encontrada em todos os Estados brasileiros onde as amostras foram coletadas, a saber: São Paulo, Bahia, Distrito Federal, Paraíba, Pernambuco, Rio Grande do Norte e Rondônia. Foram obtidos 15 isolados (30\%) de D. alata provenientes dos Estados de Pernambuco (2), de Rondônia (12) e do Rio Grande do Norte (1), e 35 isolados (70 \%) de D. cayennensis provenientes de todos esses Estados, com exceção de Rondônia (Tabela 1). A menor freqüência de isolados em $D$. alata deveu-se, provavelmente, à sua maior resistência à doença (Oliveira

TABELA 1 - Ocorrência da podridão-verde do inhame (Dioscorea spp.), causada por Penicillium sclerotigenum, em municípios de sete estados brasileiros

\begin{tabular}{|c|c|c|c|}
\hline Código do isolado & Hospedeiro & Município & Estado \\
\hline SP 2 & D. cayennensis & Piracicaba & São Paulo \\
\hline$\overline{\mathrm{BA}} 1$ & D. cayennensis & Ilhéus & Bahia \\
\hline BA 2 & D. cayennensis & Ilhéus & Bahia \\
\hline BA 14 & D. cayennensis & Jequié & Bahia \\
\hline BA 16 & D. cayennensis & Itabun a & Bahia \\
\hline BA 28 & D. cayennensis & Cruz das Almas & Bahia \\
\hline BA 30 & D. cayennensis & Cruz das Almas & Bahia \\
\hline DF 1 & D. cayennensis & Brasília & Distrito Federal \\
\hline DF 2 & D. cayennensis & Brasília & Distrito Federal \\
\hline PB 1 e PB 2 & D. cayennensis & João Pessoa & Paraíba \\
\hline PB 5 & D. cayennensis & João Pessoa & Paraíba \\
\hline PE 1 & D. cayennen sis & Vitória de Santo Antão & Pernambuco \\
\hline PE 2 & D. cayennensis & Vitória de Santo Antão & Pernambuco \\
\hline PE 3 & D. cayennensis & Vitória de Santo Antão & Pernambuco \\
\hline PE 4 & D. cayennensis & Catende & Pernambuco \\
\hline PE 5 & D. cayennensis & Catende & Pernambuco \\
\hline PE 6 & D. cayennensis & Vicênci a & Pernambuco \\
\hline PE 7 & D. cayennensis & Vicência & Pernambuco \\
\hline PE 8 & D. cayennensis & Vicência & Pernambuco \\
\hline PE 13 & D. alata & Moreno & Pernambuco \\
\hline PE 20 & D. cayennensis & Moreno & Pernambuco \\
\hline PE 22 & D. cayennensis & Moreno & Pernambuco \\
\hline PE 24 & D. cayennensis & Moreno & Pernambuco \\
\hline PE 35 & D. cayennensis & Condado & Pernambuco \\
\hline PE 36 & D. cayennensis & Condado & Pernambuco \\
\hline PE 38 & D. cayennensis & Condado & Pernambuco \\
\hline PE 39 & D. cayennensis & Catende & Pernambuco \\
\hline PE 46 & D. cayennensis & Catende & Pernambuco \\
\hline PE 47 & D. cayennensis & Catende & Pernambuco \\
\hline PE 48 & D. cayenne nsis & Condado & Pernambuco \\
\hline PE 50 & D. cayennensis & Gravatá & Pernambuco \\
\hline PE 54 & D. alata & Gravatá & Pernambuco \\
\hline RN 2 & D. cayennensis & Mossoró & Rio Grande do Norte \\
\hline RN 3 & D. cayennensis & Mossoró & Rio Grande doNorte \\
\hline RN 4 & D. alata & São José do Mipibu & Rio Grande do Norte \\
\hline RN 5 & D. cayennensis & São José do Mipibu & Rio Grande do Norte \\
\hline RN 6 & D. cayennensis & São José do Mipibu & Rio Grande do Norte \\
\hline RO 1 e RO 2 & D. alata & Porto Velho & Rondônia \\
\hline RO 4 a RO 12 & D. alata & Porto Velho & Rondônia \\
\hline RO 14 & D. alata & Porto Velho & Rondônia \\
\hline
\end{tabular}


et al., 2006a) e por ser a espécie $D$. cayennnesis mais cultivada, havendo, portanto, maior oferta no mercado. Embora as amostras não tenham sido coletadas em todos os locais onde o inhame é cultivado, os locais estudados na pesquisa representam tanto áreas produtoras quanto áreas não produtoras.

Quanto à sintomatologia da doença, não houve variação nas diversas amostras coletadas observandose sempre o crescimento micelial externo, de cor verde bem evidente, formando sinais da doença (Figura 1A). A necrose marrom interna era sempre profunda, de grande extensão lateral (Figura 1D), com aparência úmida e progressão rápida em $D$. cayennensis e restrita em $D$. alata, confirmando o observado por Oliveira et al. (2006a). Não foram observados esclerócios em túberas, apenas em meio de cultura (Figuras 1E-F). Talvez as túberas inoculadas não tenham produzido esclerócios em função da temperatura na qual foram mantidas no laboratório.
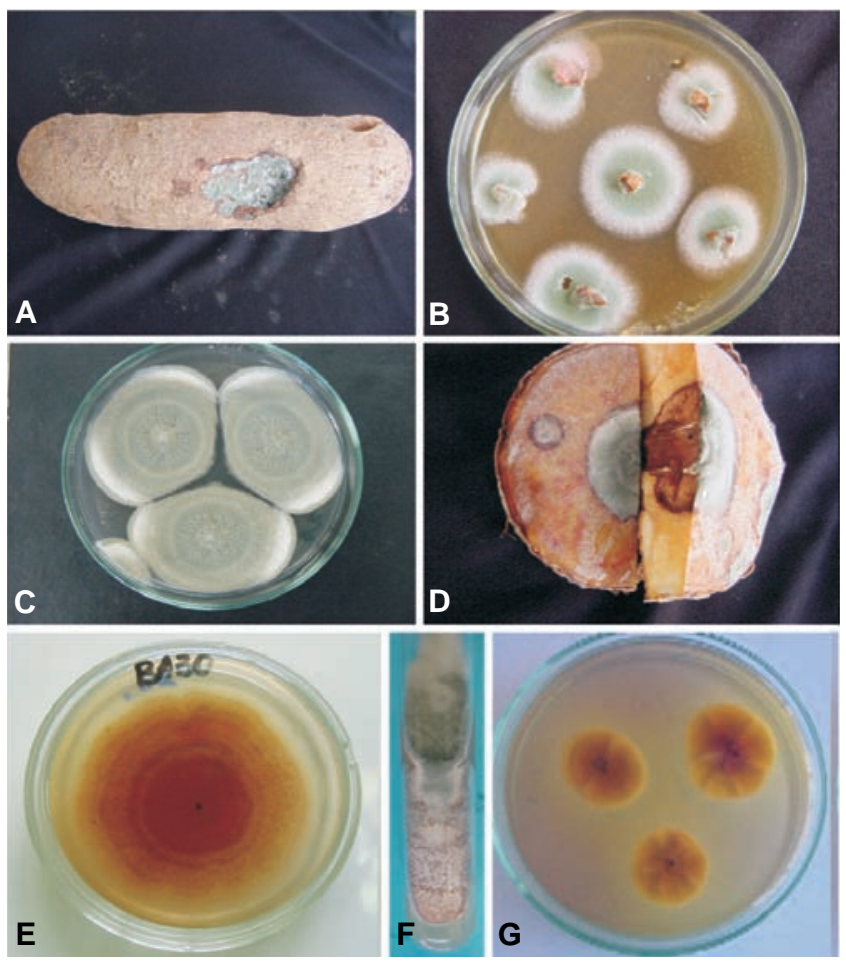

FIG. 1 - A. Túbera comercial de inhame da costa portadora de lesão natural da podridão-verde; B. Crescimento de P. sclerotigenum em meio MEA, a partir de fragmentos de inhame da costa, após isolamento; C. Colônias típicas de P. sclerotigenum em meio de cultura CYA, após sete dias, a $25^{\circ} \mathrm{C}$; D. Teste de patogenicidade positivo, mostrando o crescimento micelial do patógeno na superfície da seção da túbera comercial e a profundidade da lesão, com presença de pigmento marrom; E. Reverso da colônia, em meio BDA, mostrando intensa produção de pigmento marrom e esclerócios; F. Intensa produção de esclerócios a $30^{\circ} \mathrm{C}$, em tubo contendo CYA; G. Reverso da colônia em meio CREA a $25{ }^{\circ} \mathrm{C}$, mostrando a produção de ácido, após sete dias (área amarela circundando as colônias) .
Em associação constante com a doença, entre os 50 isolados, foi identificada unicamente $P$. sclerotigenum (Figura 1B-C), sendo todos os isolados patogênicos à espécie de inhame de origem, após inoculações em cinco seções de túberas comerciais para cada isolado (Figura 1D).

O crescimento de todos isolados de $P$. sclerotigenum nos meios de cultura CYA, MEA e YES utilizados para identificação da espécie foi considerado muito bom, com diâmetro da colônia acima de $40 \mathrm{~mm}$, aos sete dias a 25 ${ }^{\circ} \mathrm{C}$ conforme observado por Samson \& Frisvad (2004). A maioria dos isolados produziu pigmento marrom solúvel, quando em meio BDA (Figura 1E), não sendo observada produção de pigmento nos meios de cultura utilizados para a identificação de espécies de Penicillium. Vários isolados produziram ácido no meio CREA, sendo esta produção representada pelo halo amarelo ao redor da colônia (Figura $1 G)$. Não houve produção de base por nenhum dos isolados, corroborando com os resultados obtidos por Samson \& Frisvad (2004) ao estudarem o comportamento fisiológico de oito isolados de $P$. sclerotigenum, oriundos de diferentes países, não incluído o Brasil. Observou-se diversidade morfológica nos tipos de colônias dos isolados testados no meio CYA (Figura 2), independentemente da espécie de inhame e do local de origem, sendo este o primeiro relato na literatura de diversidade na aparência das colônias para $P$. sclerotigenum. Não foi observada variação na aparência das colônias entre os isolados em meio CYAS, sendo este meio o único em que $P$. sclerotigenum desenvolveu-se fracamente, confirmando Samson \& Frisvad (2004).

Os dados morfométricos obtidos com os 50 isolados confirmaram a identificação dos isolados como pertencentes à espécie $P$. sclerotigenum. Os valores médios obtidos de cada estrutura taxonômica estão apresentados na Tabela 2. Pela análise geral, comparando-se com os dados obtidos por Yamamoto et al. (1955), Moura et al. (1976) e Frsivad \& Samson (2004), conclui-se que, apesar da diversidade na aparência das colônias, os

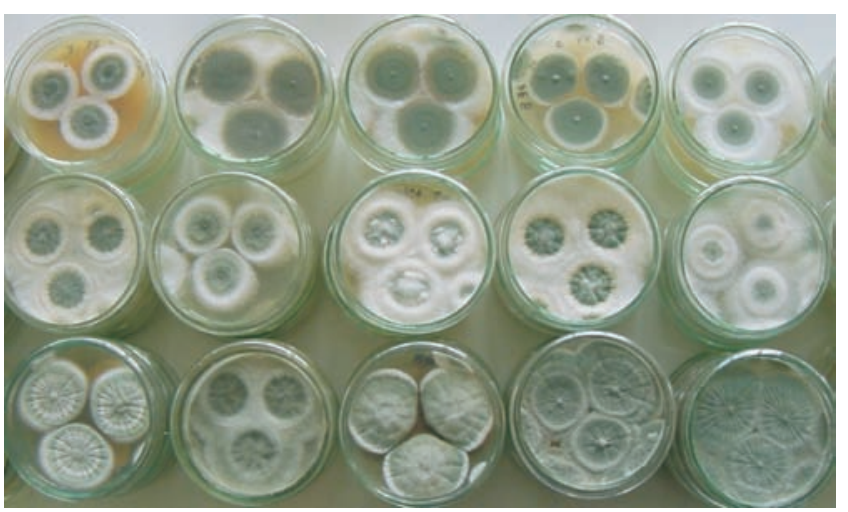

FIG. 2 - Diversidade morfológica na aparência das colônias de isolados de Penicillium sclerotigenum, provenientes de sete estados brasileiros, em meio de cultura CYA, após cultivo a $25^{\circ} \mathrm{C}$, por sete dias. 
Distribuição geográfica e diversidade morfológica de culturas...

TABELA 2 - Média morfométrica dos cinqüenta isolados de Penicillium sclerotigenum em meio CYA

\begin{tabular}{|c|c|c|c|c|c|c|c|c|c|c|}
\hline \multirow{3}{*}{$\begin{array}{l}\text { P. sclerotigenum } \\
\text { (50 isolados) }\end{array}$} & \multicolumn{10}{|c|}{ Variáveis ( em $\mu \mathrm{m}$ ) } \\
\hline & \multicolumn{2}{|c|}{ Conídio } & \multicolumn{2}{|c|}{ Fiálide } & \multicolumn{2}{|c|}{ Metula } & \multicolumn{2}{|c|}{ Ramo } & \multicolumn{2}{|c|}{ Esclerócio } \\
\hline & Comp. & largura & comp. & largura & comp. & largura & comp. & largura & comp. & largura \\
\hline Média & 4,3 & 3,4 & 10,6 & 2,8 & 13,3 & 3,0 & 20,4 & 3,2 & 207,4 & 176,1 \\
\hline Amplitude & $3,7-4,7$ & $3,1-3,8$ & $10,0-11,7$ & $2,2-3,3$ & $11,1-15,5$ & $2,6-3,3$ & $14,6-22,0$ & $2,7-3,6$ & $143,2-309,4$ & $129,3260,0$ \\
\hline Intervalo de confiança & $4,2-4,4$ & $3,3-3,4$ & $10,5-10,7$ & $2,7-2,8$ & $13,0-13,5$ & $2,9-3,0$ & $19,9-21,0$ & $3,1-3,2$ & $197,5-217,4$ & $167,4-184,7$ \\
\hline Desvio-padrão & 0,3 & 0,2 & 0,3 & 0,3 & 0,8 & 0,2 & 1,6 & 0,2 & 36,2 & 31,6 \\
\hline Coeficiente de variação & 5,7 & 5,8 & 2,7 & 9,1 & 6,3 & 5,3 & 8,0 & 5,3 & 17,4 & 18,0 \\
\hline Frisvad \& Samson, 2004 & $4,0-5,0$ & $2,5-3,5$ & $8,0-12,0$ & $2,5-3,0$ & $13,0-22,0$ & $3,0-4,0$ & $17,0-25,0$ & $3,0-4,0$ & $150,0-300,0$ & $\mathrm{XXXXXX}$ \\
\hline Moura et al., 1976 & $4,0-6,0$ & $2,9-3,9$ & $7,0-12,0$ & $2,7-3,0$ & $9,0-17,0$ & $2,7-3,8$ & XXXXXX & XXXXX & $161,0-376,0$ & $160-250,0$ \\
\hline Yamamoto et al., 1995 & $4,0 \quad 6,0$ & $3,0 \quad 4,0$ & $8,0 \quad 11,2$ & $2,5 \quad 3,5$ & $9,0 \quad 16,0$ & $2,5 \quad 4,0$ & XXXXXX & XXXXX & $170,0 \quad 380,0$ & 160320,0 \\
\hline
\end{tabular}

valores micrométricos médios das estruturas taxonômicas dos diferentes isolados foram consistentes, com baixos coeficientes de variação e estavam de acordo com os dos autores supracitados.

A produção de esclerócios nos meio CYA ocorreu à temperatura de $30{ }^{\circ} \mathrm{C}$ e não a $25^{\circ} \mathrm{C}$, sendo mais intensa nas culturas em tubo inclinado contendo meio CYA (Figura $1 \mathrm{~F}$ ) do que em placa de Petri, contrariando o que afirmaram Samson \& Frisvad (2004) quanto à temperatura de indução de produção de esclerócios. Após a identificação dos isolados de Penicillium sp., verificouse que a única espécie presente como agente etiológico da podridão-verde foi $P$. sclerotigenum, independentemente da espécie de inhame e da origem da amostra. Não foram, portanto, assinaladas outras espécies em associação com a podridão-verde, em contraste aos relatos na Nigéria por Ogundana et al. (1970) e Adeniji (1970).

É importante a determinação da distribuição de $P$. sclerotigenum no Brasil, pois, além das perdas naturais impostas à cultura do inhame, esse patógeno causa também podridão em outras culturas, a exemplo de maçã, pêra e banana, conforme demonstraram Oliveira et al. (2006b). Assim, aumenta a possibilidade de alimentos, além dos já mencionados, estarem contaminados com patulina, micotoxina também produzida por $P$. sclerotigenum, constatada, até o momento, em meio de cultura (Samson \& Frisvad, 2004). A patulina pode induzir no homem sintomas agudos (convulsão, edema, náusea, ulceração e inflamação intestinal) e supostamente crônicos (neurotoxicidade, imunosupressão, teratogenicidade e carcinogenicidade) (Moake et al., 2005). Pela importância do assunto, novas pesquisas estão programadas pelo grupo para detecção de patulina em inhame.

\section{AGRADECIMENTOS}

Os autores agradecem ao $\mathrm{CNPq}$ pelo financiamento do trabalho e a Profa. Maria José dos Santos Fernandes da Micoteca URM, UFPE, Recife, responsável pela confirmação da identificação de espécies de Penicillium a serem depositados nesta micoteca.

\section{REFERÊNCIAS BIBLIOGRÁFICAS}

ADENIJI, M.O. Fungi associated with storage decay of yams in Nigeria. Phytopathology 60:590-592. 1970.

FIGUEIREDO, M.B. Métodos de preservação de fungos patogênicos. Biológico 63:73-82. 2001.

MENEZES, M. \& ASSIS, S.M.P. Guia prático para fungos fitopatogênicos. $2^{\text {a }}$. Ed. Recife PE. Imprensa Universitária, UFRPE, 2004.

MOAKE, M.M., PADILLA-ZAKOUR, O.I. \& WOROBO, R.W. Comprehensive review of patulin control methods in foods. Comprehensive Reviews in Food Science and Food Safety 1:8-21. 2005.

MOURA, R.M. Doenças do Inhame-da-costa (Dioscorea cayennensis). In: Kimati, H., Amorim, L., Resende, J.A.M., Bergamim Filho, A. \& Camargo, L.F.A. (Eds.) Manual de Fitopatologia. Vol. 2. Doenças das Plantas Cultivadas. São Paulo SP. Editora Ceres. 2005.

MOURA, R.M., RIBEIRO, G.P., COELHO, R.S.B. \& SILVA JÚNIOR, J.N. Penicillium sclerotigenum Yamamoto, principal fungo causador de podridão em túberas de inhame (Dioscorea cayennensis Lam.), no Estado de Pernambuco, Brasil. Fitopatologia Brasileira 1:67-78. 1976.

OGUNDANA, S.K., NAQIV, S.H.Z. \& EKUNDAYO, J.A. Fungi associated with soft-rot of yams (Dioscorea spp.) in storage in Nigeria. Transactions British Mycology Society 54:445-451. 1970.

OLIVEIRA, I.S., MOURA, R. M., LUZ, E.D.M.N., BEZERRA, J.L., TORRES, G.R.C. \& MAIA, L.C. Severidade da podridãoverde em inhames e especialização fisiológica em Penicillium sclerotigenum. Fitopatologia Brasileira 31:94-98. 2006a.

OLIVEIRA, I.S., LUZ, E.D.M.N., MOURA, R.M. \& MAIA, L.C. Patogenicidade de Penicillium sclerotigenum a diferentes 
frutas e hortaliças em pós-colheita. Fitopatologia Brasileira 31:408-410. 2006b.

SAMSON, R.A. \& FRISVAD, J.C. Penicillium subgenus Penicillium: new taxonomic schemes and mycotoxins and other extrolites. Studies in Mycology (Baarn) 49. 2004.

SANTOS, E.S. Inhame (Dioscorea spp.). Aspectos Básicos da Cultura. João Pessoa PB. Empresa Estadual de Pesquisa
Agropecuária da Paraíba. 1996.

SANTOS, E.S. Cultura do inhame (Dioscorea sp.). João Pessoa PB. Empresa Estadual de Pesquisa Agropecuária da Paraíba. 2002.

YAMAMOTO, W., YOSHITANI, K. \& MAEDA, M. Studies on Penicillium and Fusarium rot of chinese yam and their control. The Science Reports of the Hyogo University of Agriculture 2:69-79. 1955.

Recebido 11 Abril 2005 - Aceito 2 Abril 2007 - FB 5089 